\title{
Increased knowledge of Francisella genus diversity highlights the benefits of optimised DNA-based assays
}

\author{
Jon Ahlinder ${ }^{{ }^{* \dagger}}$, Caroline Öhrman ${ }^{1 \dagger}$, Kerstin Svensson ${ }^{1}$, Petter Lindgren ${ }^{1}$, Anders Johansson ${ }^{2,3}$,
} Mats Forsman ${ }^{1}$, Pär Larsson ${ }^{1}$ and Andreas Sjödin ${ }^{1}$

\begin{abstract}
Background: Recent advances in sequencing technologies offer promising tools for generating large numbers of genomes, larger typing databases and improved mapping of environmental bacterial diversity. However, DNA-based methods for the detection of Francisella were developed with limited knowledge about genetic diversity. This, together with the high sequence identity between several Francisella species, means there is a high risk of false identification and detection of the highly virulent pathogen Francisella tularensis. Moreover, phylogenetic reconstructions using single or limited numbers of marker sequences often result in incorrect tree topologies and inferred evolutionary distances. The recent growth in publicly accessible whole-genome sequences now allows evaluation of published genetic markers to determine optimal combinations of markers that minimise both time and laboratory costs.
\end{abstract}

Results: In the present study, we evaluated 38 previously published DNA markers and the corresponding PCR primers against 42 genomes representing the currently known diversity of the genus Francisella. The results highlight that PCR assays for Francisella tularensis are often complicated by low specificity, resulting in a high probability of false positives. A method to select a set of one to seven markers for obtaining optimal phylogenetic resolution or diagnostic accuracy is presented.

Conclusions: Current multiple-locus sequence-typing systems and detection assays of Francisella, could be improved by redesigning some of the primers and reselecting typing markers. The use of only a few optimally selected sequence-typing markers allows construction of phylogenetic topologies with almost the same accuracy as topologies based on whole-genome sequences.

Keywords: Bacterial-typing techniques, Optimisation, Francisella, Metagenomics, Phylogeny, Assay, Diversity, $N G S, P C R$

\section{Background}

The gram-negative pathogen Francisella tularensis is the causative agent of tularemia and is classified as a category-A biological-threat agent [1]. Natural transmission of tularemia to humans is complex, occurring via the inhalation of infective aerosols, ingestion of contaminated water, handling sick or dead animals, ingestion of

\footnotetext{
* Correspondence: jon.ahlinder@foi.se

${ }^{\dagger}$ Equal contributors

'Division of CBRN Security and Defence, FOI, Swedish Defence Research Agency, SE- 906 21, Umeå, Sweden

Full list of author information is available at the end of the article
}

infected food-stuffs, or bites of infected arthropods such as ticks, biting flies or mosquitoes [2].

The genus Francisella includes a number of closely related but ecologically distinct species that can be divided into two main genetic clades [3]. These bacteria exhibit a large variety of lifestyles, including specialised intracellular pathogens of mammals (F. tularensis subsp. tularensis and subsp. holarctica) and fish (F. noatunensis), Francisella-like endosymbionts (FLEs) (represented here by Wolbachia persica) and freely living generalists (F. philomiragia x F. novicida) causing disease predominantly in humans with a compromised immune defense

\section{Biomed Central}


[4]. The taxonomic boundaries of Francisella have recently been debated, in particular for F. novicida [5,6]. Recent breakthroughs in sequencing techniques have enabled public access to whole-genome sequences that can shed light on previously unknown diversity within the Francisella genus. The mode of genetic inheritance varies within the genus: the overall recombination rate is $34 \%$ of the genes within the Francisella core genome, although recombination is virtually non-existent in F. tularensis and F. noatunensis [3,7]. These ecological and reproductive differences which lead to genetic diversity make Francisella an ideal choice for evaluation of diagnostic PCR-based DNA markers and developing sample sequencing methods for phylogenetic analyses.

Over the last decade, PCR methods have been successfully applied for the rapid identification and classification of Francisella isolates [8]. An obvious drawback with DNA-based approaches is the possibility of crossreactivity with non-pathogenic but closely related Francisella subspecies occurring naturally in the environment $[3,9,10]$. This could distract biological surveillance systems, such as the BioWatch Program [11], and give false-positive alarms $[12,13]$. Therefore, primer pairs need to be defined so that an unknown isolate is identified and attributed to the correct species or subspecies. Previously published sequence markers designed for identification or detection of Francisella have been developed without taking into consideration the current knowledge of genetic diversity of the genus, in particular the recently discovered species $F$. noatunensis and F. hispaniensis.

The specificity of Francisella detection assays has often been controlled by testing reactivity with non-Francisella bacterial species. Typically, no other species besides $F$. tularensis (including subspecies tularensis, mediasiatica and holarctica), F. novicida and F. philomiragia have been included as representatives of the Francisella genus [14-17]. As with PCR detection, current knowledge on the diversity of the Francisella genus affects the choice of genetic markers used for obtaining true phylogenetic trees by PCR-based sequence-typing analysis. For $F$. tularensis, multi-locus typing schemes targeting overlapping, as well as separate, genes have been described $[18,19]$. However, the resolution was limited, allowing discrimination of only the major genetic clades of the species. Recent advances in sequencing and the increased availability of publicly accessible genomic sequences have enabled phylogenetic trees obtained by analysing sequence markers to be evaluated. Whole-genome sequencing is not always desirable for large bacterial sample sets, as such analysis normally generates large amount of data which requires substantial increase in labour and time. Therefore, multiplexed target amplification of selected genomic regions using next generation sequencing (NGS) have recently been proposed [20,21].
A considerable effort in the study of bacterial pathogens has been devoted to evaluating alternative evolutionary histories by comparing topologies [22-25]. In order to facilitate these comparisons, various topological distance metrics have been proposed, such as the Robinson-Foulds (RF) or symmetric distance [26], branch-score distance [27], path-length metrics [28] and nearest-neighbour interchanging [29]. To quantify similarity, all these metrics focus on topological features (order of nodes within the topology) and/or branch- or path-length differences (between nodes and leaves). An alternative approach would be to construct and test a parameter describing the degree of incompatibility (i.e. conflicting phylogenetic signals) between topologies. To the best of our knowledge, no such straightforward metric exists for this particular purpose of quantifying the level of incompatibility. Alternative topologies could be compared with a reference topology obtained from, e. g. the literature, a large set of concatenated genes or a source of high-quality whole-genome data. Ideally, such reference topology should mimic the species phylogeny as accurate as possible.

In this study, we evaluated the specificity of detection and classification of Francisella by first comparing published PCR primers against whole-genome sequences representing the known diversity of the genus. Second, we examined the sequence-marker robustness and resolution by comparing different sets of one to seven markers using a modified version of the RF metric. Finally, we showed that optimal sets of markers outperform other combinations with respect to phylogenetic robustness and resolution.

\section{Results}

\section{Overall fit between DNA-markers and whole-genome sequences of Francisella}

A total of 42 publicly available Francisella genome sequences were screened for sequences (Table 1) of 38 published markers (Table 2). 14 markers had incomplete sets of marker sequences (Figure 1). The lack of $16 \mathrm{~S}$ marker sequences in FSC022, FSC033, MA002987, GA993549, and GA993548 was probably due to the low quality of the genome sequences, which were all sequenced with early versions of 454 sequencing technology. The lack of sequences for the remaining 10 markers was most likely because they were designed for real-time PCR molecular detection or possibly due to uncovered regions in the sequence (Additional file 1).

The primer specificities of the 38 DNA markers were calculated, resulting in scores ranging from 0 to 7.2 (Figure 1). Importantly, the calculation was performed for Francisella species besides those included in the publication from which the marker originated. A primer score of zero represented a perfect match without any 
Table 1 Genomes sequences included in the study

\begin{tabular}{|c|c|c|}
\hline Species & ID & BioProject ID \\
\hline F. tularensis subsp. holarctica & FSC200 & 16087 \\
\hline F. tularensis subsp. holarctica & FSC208 & 73467 \\
\hline F. tularensis subsp. holarctica & RC503 & 30637 \\
\hline F. tularensis subsp. holarctica & LVS & 16421 \\
\hline F. tularensis subsp. holarctica & FSC539 & 73393 \\
\hline F. tularensis subsp. holarctica & OR96-246 & 30669 \\
\hline F. tularensis subsp. holarctica & FTA & 20197 \\
\hline F. tularensis subsp. holarctica & URFT1 & 19645 \\
\hline F. tularensis subsp. holarctica & MI00-1730 & 30635 \\
\hline F. tularensis subsp. holarctica & OSU18 & 17265 \\
\hline F. tularensis subsp. holarctica & FSC021 & 73369 \\
\hline F. tularensis subsp. holarctica & FSC022 & 19015 \\
\hline F. tularensis subsp. mediasiatica & FSC147 & 19571 \\
\hline F. tularensis subsp. mediasiatica & FSC148 & 73379 \\
\hline F. tularensis subsp. tularensis & FSC054 & 73375 \\
\hline F. tularensis subsp. tularensis & ATCC6223 & 30629 \\
\hline F. tularensis subsp. tularensis & FSC033 & 19017 \\
\hline F. tularensis subsp. tularensis & MA00-2987 & 30443 \\
\hline F. tularensis subsp. tularensis & FSC198 & 17375 \\
\hline F. tularensis subsp. tularensis & SCHUS4 (FSC237) & \\
\hline F. novicida & FTE & 30119 \\
\hline F. novicida & U112 & 16088 \\
\hline F. novicida & FTG & 30447 \\
\hline F. novicida & GA99-3549 & 19019 \\
\hline F. novicida & FSC160 & 73385 \\
\hline F. novicida & FSC159 & 73383 \\
\hline F. novicida & GA99-3548 & 19573 \\
\hline F. hispaniensis & FSC454 & 73391 \\
\hline Wolbachia persica & FSC845 & 73171 \\
\hline F. noatunensis subsp. orientalis & FSC770 & 73389 \\
\hline F. noatunensis subsp. orientalis & FSC771 & 73447 \\
\hline F. noatunensis subsp. noatunensis & FSC846 & 73463 \\
\hline F. noatunensis subsp. noatunensis & FSC769 & 73397 \\
\hline F. noatunensis subsp. noatunensis & FSC774 & 73457 \\
\hline F. noatunensis subsp. noatunensis & FDC178 & 73465 \\
\hline F. noatunensis subsp. noatunensis & FSC772 & 73449 \\
\hline F. philomiragia & FSC154 & 73381 \\
\hline F. philomiragia & FSC145 & 73377 \\
\hline F. philomiragia & ATCC25015 & 32411 \\
\hline F. philomiragia & FSC037 & 73371 \\
\hline F. philomiragia & FSC039 & 73373 \\
\hline F. philomiragia & ATCC25017 & 27853 \\
\hline
\end{tabular}

Francisella genomes included in this study selected to represent the known diversity of Francisella: 22 strains representing the public health perspective of $F$. tularensis (clade 1 ) and 13 strains of $F$. noatunensis and F. philomiragia (clade 2) representing a fish farming industry and health perspective. mispriming events or gaps, while the maximal score of 7.2 corresponded to two mismatches in the 3 ' region and a gap of 10 bases within the region targeted by a primer (see marker 21-ISFtu2). All primer scores are presented in Figure 1 and summarised in Table 2. The limit for possible amplification was assumed to be a score value of two, in agreement with the NCBI Primer-BLAST default primer specificity stringency setting. Scores below two $(<2)$ are denoted as low score and score above two $(\geq 2)$ are denoted as high score [30].

\section{Evaluation of DNA markers}

The marker 01-16S [14] targeting 16S rRNA was the only marker with a low score $(<1)$ for all the investigated genomes. A total of nine markers (01-16S, 03-16S-Itr-23S, 04-16S-Itr-23S, 08-fabH, 18-groEL 23-lpnA, 25-mdh, 30prfb and 35-tpiA) had scores $<2$ in all subspecies. However, some of these markers, e.g. 23-lpnA, showed a clear difference in scores between clade 1 and clade 2, as clade 1 yielded almost perfect matches, while scores in clade 2 were always $>1$.

Most of the included primers amplified sequences of $F$. tularensis (including subspecies tularensis, mediasiatica, and holarctica) and F. novicida of clade 1 and less frequently amplified sequences of $F$. noatunensis and $F$. philomiragia, of clade 2. Fifteen markers (05-aroA, 07-dnaA, 11-fopA-in, 12-fopA-out, 13-fopA, 14-FtM19, 15-FtM19, 19-iglC, 22-lpnA, 26-mutS, 27-parC, 31-putA, 36-tpiA, 37-trpE and 38-uup) gave low scores for clade 1 and high scores for clade 2. Marker 38-uup also had low scores in one isolate of philomiragia, and the marker 19iglC had low scores in $F$. noatunensis subsp. orientalis and in two isolates of $F$. philomiragia.

Of these fifteen markers, twelve (05-aroA, 07-dnaA, 12-fopA-out, 13-fopA, 19-iglC, 22-lpnA, 26-mutS, 27-parC, 31-putA, 36-tpiA, 37-trpE and 38-uup) had low scores for F. hispaniensis FSC454 and/or W. persica FSC845 as well as low scores in clade 1. Only three (11-fopA-in, 14-Ft-M19 and 15-Ft-M19) out of the fifteen markers consistently differentiated clade 1 from the rest of the Francisella genus.

The marker 10-fopA was the only marker completely specific for clade 2 and only marker 24-lpnB was specific for $F$. noatunensis. Both of these exhibited lower specificity for $F$. noatunensis subsp. orientalis genomes.

Several markers displayed complex amplification patterns. Seven markers (02-16S-Itr-23S, 06-atpA, 09-fopA, 29-pgm, 32-rpoA, 33-rpoB, 34-sdhA) had high scores in one or more species or subspecies, e.g. the marker 09fopA had a low score in all included strains except in $F$. hispaniensis FSC454 and W. persica FSC845. Similar results were observed for 02-16S-Itr-23S, 29-pgm, 33rpoB and 34-sdhA. 
Table $2 \mathrm{~A}$ list of the markers selected to represent published DNA-based markers for molecular PCR detection or phylogenetic identification targeting Francisella

\begin{tabular}{|c|c|c|c|c|}
\hline $\begin{array}{l}\text { Marker name/ } \\
\text { Target gene }\end{array}$ & Gene locus_tag ${ }^{a}$ & $\begin{array}{l}\text { Amplicon } \\
\text { size (bp) }\end{array}$ & Genomic location $^{a}$ & Reference \\
\hline $01-165$ & FTT_r04, FTT_r07, FTT_r10 & 1139 & 1311156-2294, 1378275-9413, 1771610-2748 & {$[17,37,38,56]$} \\
\hline $02-16 s+1 t s+23 s$ & FTT_r04, FTT_r07, FTT_r10 & 915 & 1311470-2371, 1378876-9490, 1771911-2825 & {$[34]$} \\
\hline $03-16 s+1 t s+23 s$ & $\begin{array}{l}\text { FTT_r03-FTT_r04, FTT_r06-FTT_r07, } \\
\text { FTT_r09-FT_r10 }\end{array}$ & 948 & 1310519-1466, 1377638-8585, 1770973-1920 & [34] \\
\hline $04-16 s+1 t s+23 s$ & FTT_r03, FTT_r06, FTT_r09 & 925 & 1309613-10537, 1376732-7656, 1770067-991 & {$[34]$} \\
\hline 05-aroA & FTT_0588 & 650 & $608150-799$ & {$[18,61]$} \\
\hline 06-atpA & FTT_0062 & 634 & $62762-3395$ & {$[18,61]$} \\
\hline 07-dnaA & FTT_0001 & 618 & $303-920$ & [19] \\
\hline 08-fabH & FTT_1373 & 1289 & $1418892-20155$ & [62] \\
\hline 09-fopA & FTT_0583 & 886 & 599105-990 & [19] \\
\hline 10-fopA & FTT_0583 & 1068 & $599148-600215$ & {$[34]$} \\
\hline 11-fopA-in & FTT_0583 & 404 & 599526-929 & [15] \\
\hline 12-fopA-out & FTT_0583 & 708 & $599428-600135$ & [15] \\
\hline 13-fopA & FTT_0583 & 86 & $599767-852$ & {$[9,16]$} \\
\hline 14-FtM19 & FTT_1472C & 250 & $1524132-381$ & {$[56,58]$} \\
\hline 15-FtM19 & FTT_1472C & 316 & $1524066-381$ & [65] \\
\hline 16-FTT0376 & FTT_0376 & 107 & $377718-824$ & {$[17]$} \\
\hline 17-FTT0523 & FTT_0523 & 91 & $546620 . .712$ & {$[17]$} \\
\hline 18-groEL & FTT_1696 & 803 & $1764659-5461$ & [34] \\
\hline 19-iglC & FTT_1712C & 84 & $1792514-597$ & {$[9,16]$} \\
\hline 20-ISFtu2 ${ }^{\mathrm{b}}$ & FTT_1311 & 390 & $1335726-6115$ & {$[56,59]$} \\
\hline 21-ISFtu2 & FTT_0099 & 97 & $103438-534^{c}$ & {$[9,16]$} \\
\hline 22-IpnA ${ }^{b}$ & FTT_0901 & 407 & $909857-10263$ & {$[19,37,38,56,57]$} \\
\hline 23-IpnA & FTT_0901 & 93 & $910211-301$ & {$[9,16]$} \\
\hline 24-IpnB & FTT_0904 & 252 & 911795-2046 & [34] \\
\hline 25-mdh & FTT_0535c & 715 & $556932-7646$ & {$[63,64]$} \\
\hline 26-muts & FTT_1499 & 495 & $1553224-3718$ & [19] \\
\hline 27-parC & FTT_0396 & 643 & 397063-705 & {$[18,61]$} \\
\hline 28-pdpD & FTT_1360c, FTT_1715c & 136 & $1403503-638,1796838-973$ & {$[56,60]$} \\
\hline 29-pgm & FTT_0414 & 650 & $425033-682$ & {$[18,61]$} \\
\hline 30-prfB & FTT_0191 & 376 & 207686-8061 & [19] \\
\hline 31-putA & FTT_1150c & 415 & $1165411-825$ & [19] \\
\hline 32-rрoA & FTT_0350, FTT_1442C & 914 & $349619-50532$ & {$[64]$} \\
\hline 33-rрoB & FTT_0144 & 262 & $156309-570$ & [34] \\
\hline 34-sdhA & FTT_0074 & 223 & $75065-287$ & [34] \\
\hline 35-tpiA & FTT_0080 & 484 & $83679-4162$ & [19] \\
\hline 36-tpiA & FTT_0080 & 559 & $83657-4215$ & {$[18,61]$} \\
\hline 37-trpE & FTT_1802C & 517 & 1888928-9444 & {$[18,61]$} \\
\hline 38-uup & FTT_0445 & 645 & $459229-873$ & {$[18,61]$} \\
\hline
\end{tabular}

amplicon locus tag, length and location in genome of F. tularensis strain SCHU S4.

${ }^{b}$ Primer sequence of primer Tuf1705 in marker 20-ISFtu2 and TUL-435 in marker 22-IpnA seem to be incorrectly specified in [56]. See [37] and [59] for the correct primer sequences.

${ }^{c}$ Insertion element present in multiple copies in reference. Only first position and gene specified. 


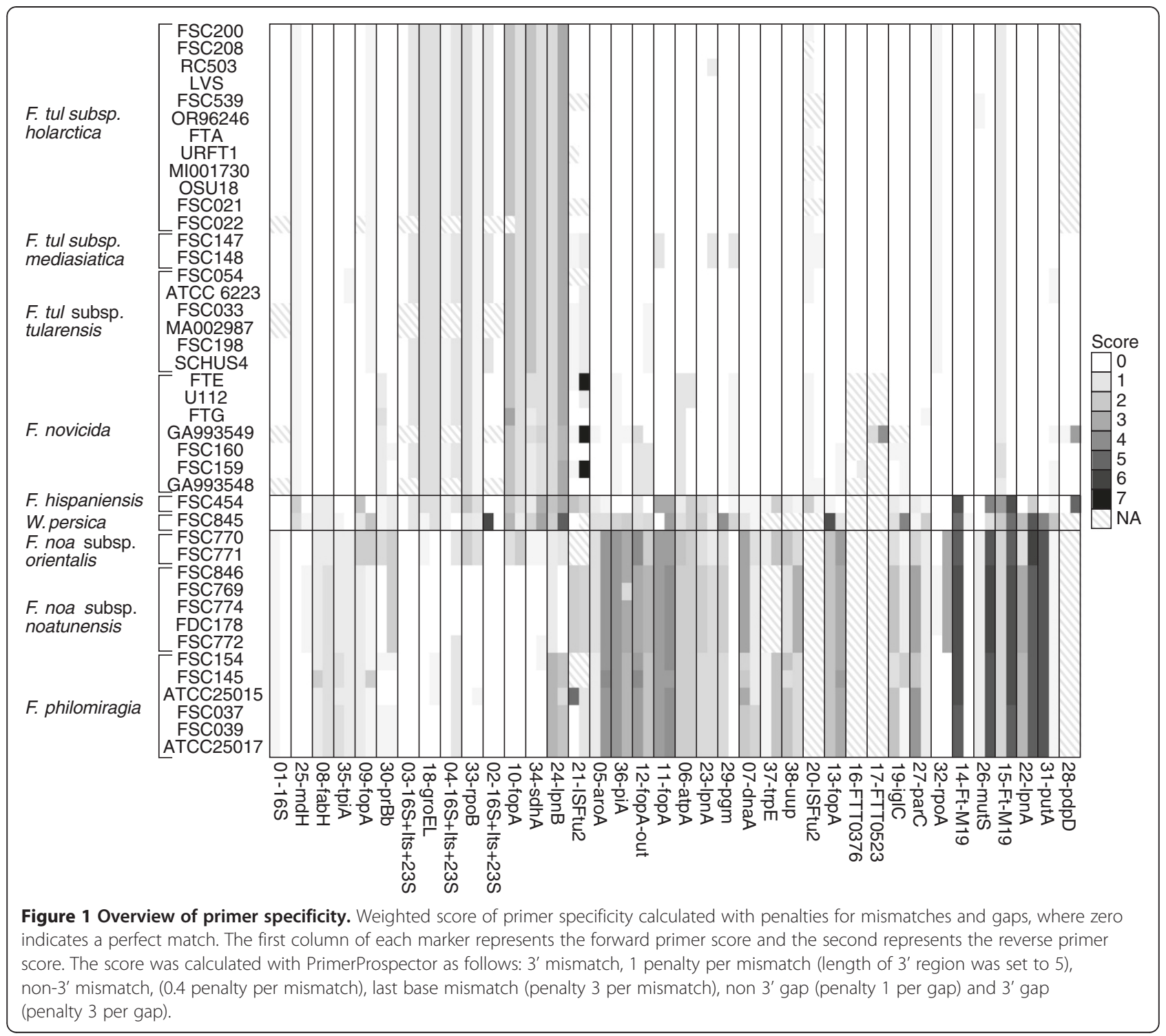

Four detection markers (16-FTT0376, 17-FTT0523, 20 ISFtu2 and 28-pdpD) had missing data (i.e. the sequence could not be found in the genome) for all clade 2 isolates plus $W$. persica. The markers 16-FTT0376 and 17FTT0523 had missing sequences for $F$. hispaniensis and $F$. tularensis subsp. novicida, except the isolates FSC159 and GA993549, respectively. The marker 21-ISFtu2 had missing sequences as well as mismatches in almost all subspecies represented. A summary of the DNA-marker evaluation can be found in Table 3, and more detailed information, including earlier published results for each marker, can be found in Additional file 1.

\section{Evaluation of sample-sequencing approaches for phylogenetic analyses}

In the phylogenetic comparison analysis, we focused not only on the entire Francisella genus, but also analysed clades 1 and 2 separately. These sub-populations exhibit different lifestyles and environmental niches and are therefore of interest to different scientific fields $[3,7,18]$. The differences between the poorest and best resolved single marker topologies of the entire genus compared to the whole-genome reference topology (Figure 2) are highlighted in Figure 3A-C. All topologies are shown in Additional file 2. The parameter estimates of the phylogenetic analysis are summarised in Additional file 3. In general for the analysis of the entire genus, the optimal substitution model was parameter rich, i.e. typically the generalised time-reversible (GTR) [31] or HasegawaKishino-Yano (HKY85) [32] models with either invariant sites parameter $(\alpha)$ or rate heterogeneity over sites $(I)$. Moderate or even low parameter-rich substitution models were favoured in the separate clade analyses, in particular for clade 1, where Jukes-Cantor (JC) [33] or 
Table 3 Summary of estimated amplification performance of primer pairs representing published DNA-based markers targeting Francisella

\begin{tabular}{|c|c|}
\hline Estimated amplification performance & Marker id \\
\hline Amplifies the entire genus & $\begin{array}{l}\text { 01-16S, 03-16S-Itr-23S, 04-16S-Itr-23S, 08-fabH, 18-groEL, 23-IpnAa, } \\
\text { 25-mdh, 30-prfb and 35-tpiA. }\end{array}$ \\
\hline Amplifies clade 1 but not clade 2 & 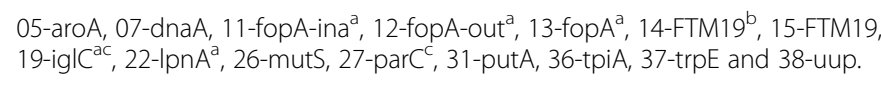 \\
\hline Amplifies clade 1 but no other Francisella species. & 11 -fopA-in ${ }^{\mathrm{a}}, 14-\mathrm{FtM} 19$ and $15-\mathrm{FtM} 19^{\mathrm{a}}$ \\
\hline Amplifies clade 1 as well as F. hispaniensis and W. persica & 05-aroA, 07-dnaA, 12-fopA-out ${ }^{\mathrm{a}}$, 27-parC ${ }^{\mathrm{C}}$ and 36-tpiA. \\
\hline Amplifies clade 1 as well as F. hispaniensis & 13-fopA $A^{a}$, 19-iglC, $22-$ IpnA, 31-putA, 37-trpE and 38-uup. \\
\hline Amplifies clade 1 as well as $W$. persica & 26-muts \\
\hline Amplifies clade 2 but not clade 1 & 10-fopA \\
\hline Amplifies noatunensis but not the other species & 24-IpnB \\
\hline Amplifies all isolates except some certain species. & 02-16S-Itr-23S, 06-atpA, 09-fopA, 29-pgm, 32-rpoA, 33-rpoB and 34-sdhA. \\
\hline Amplifies all except $F$. hispaniensis and W. persica & 09-fopA \\
\hline Amplifies all except $F$. hispaniensis & 33-rрoB \\
\hline Amplifies all except $F$. tularensis, W. persica and F. hispaniensis & $34-s d h A$ \\
\hline Amplifies all except $W$. persica & 02-16S-Itr-23S, 29-pgm \\
\hline Amplifies all except $F$. noatunensis subsp. orientalis & 06-atpA \\
\hline Amplifies all except $F$. noatunensis & 32-rpoA \\
\hline Markers with data missing for clade 2 and W. persica & 16-FTT0376 ${ }^{\mathrm{a}}, 17-\mathrm{FTT} 0523^{\mathrm{a}}, 20-1$ SFtu2 ${ }^{\mathrm{b}}$ and 28-pdpD ${ }^{\mathrm{b}}$. \\
\hline Amplifies only F. tularensis (only when including the probe). & 16-FTT0376 and 17-FTT0523 \\
\hline $\begin{array}{l}\text { Amplifies F. tularensis subsp. mediasiatica, F. tularensis subsp. } \\
\text { holarctica and } 6 / 7 \text { F. tularensis subsp. novicida. }\end{array}$ & $28-p d p D^{b}$ \\
\hline Amplifies isolates from all clade 1 species as well as W. persica. & 20-ISFtu2 ${ }^{\mathrm{b}}$ \\
\hline $\begin{array}{l}\text { Marker with missing sequences as well as mismatches in almost } \\
\text { all subspecies represented. }\end{array}$ & 21-ISFtu2 ${ }^{\mathrm{a}}$ \\
\hline
\end{tabular}

Successful amplification was defined as having a primer score below two in both the forward and reverse primers.

${ }^{a}$ Have associated TaqMan probe which is not considered here. ${ }^{b}$ Detection by variable-length amplicon which is not considered here.

'Score of F.noatunensis subsp orientalis $<2$.

HKY85 models were found to be the optimal choice without $\alpha$ or $\Gamma$. For clade 2, it was important to include the proportion of invariant sites parameter in the analyses, because of detected recombination events [3].

Throughout the study, to facilitate the phylogeny comparisons, we made use of two metrics: degree of incongruence (inc) and difference in resolution (res). The two topologies compared were the reference topology, obtained from whole genome data, and the singlesequence or the concatenated marker sequences topology. Results from comparing single-sequence topologies against the reference phylogeny based on whole-genome sequences are summarised in Additional file 4. The comparisons varied in inc, and sometimes considerably so. In the analysis of the entire genus, the 37-trpE topology did not exhibit any incongruence compared to the reference (inc $=0$ ), although the resolution was poor. For other markers, such as 08-fabH, 27-parC, 03-16 s + ItS + 23 s, 04-16 s + ItS + 23 s, 25-mutS and 36-tpiA, the topology comparisons indicated few mismatched bipartitions (inc $<0.25$ ), whereas the opposite result was found for
11-fopA-in, 29-pgm and 30-prfB (inc > 0.35). As expected, for some single-marker topologies, particularly those with the lowest inc scores, the $\mathrm{SH}$ test did not reject congruence compared to the reference phylogeny.

Separate clade 1 topologies exhibited a lower average incongruence than topologies of the entire genus $\left(\right.$ inc $_{\text {clade } 1}=$ 0.139 vs. inc $_{\text {genus }}=0.258, p=6.6 \mathrm{e}-05$ ) and clade 2 topologies $\left(\right.$ inc $_{\text {clade1 }}=0.139$ vs. inc clade2 $\left.=0.238, p=0.0149\right)$. In several cases, clade 1 topologies were totally congruent with no mismatched bipartitions. Some of these topologies were also congruent in clade 2: $(01-16 \mathrm{~S}, 03-16 \mathrm{~s}+\mathrm{ItS}+23 \mathrm{~s}$, 04$16 \mathrm{~s}+\mathrm{ItS}+23 \mathrm{~s}, 07-$ dnaA, 08-fabH, 22-lpnA, 24-lpnB, 25mdh, 27-parC, 30-prfB, 31-putA, 35-tpiA, 36-tpiA, 37-trpE and 38-uup). The low level of incongruence was verified by the results of the SH-test, which showed that congruence in the topology comparisons could not be rejected with the exception of 19-iglC. Reported incongruences in clade 1 mostly occurred in $F$. novicida. Most assignments deviating from the reference in clade 2 were due to misplacements of subspecies $F$. philomiragia and $F$. noatunensis subsp. noatunensis. 


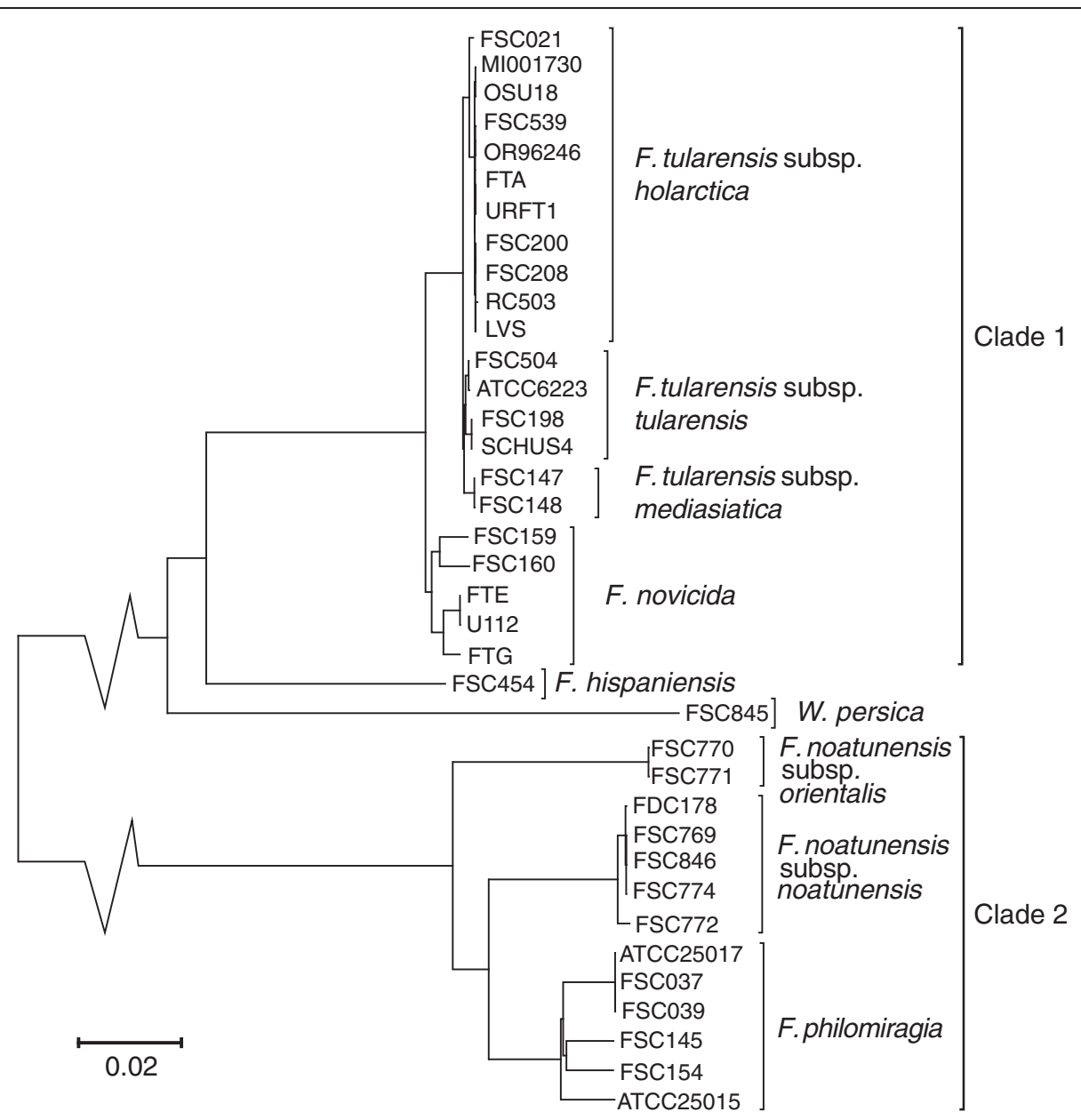

Figure 2 Whole-genome SNP phylogeny. The whole-genome phylogeny for 37 Francisella strains obtained with model averaging implemented in jModelTest using PhyML software. The removed part of the branches connecting clade 1 and 2 covers a genetic distance of 0.03 .

In the separate analysis of clade 1 , most strains not assigned according to the reference were due to poor resolution, notably topologies of markers 32-rpoA, 37trpE, 25-mdh, 24-lpnB and 19-iglC. The average resolution (res) in topologies of clade 1 was significantly higher than clade $2\left(\operatorname{res}_{\text {clade } 1}=0.723\right.$ vs. $\operatorname{res}_{\text {clade } 2}=0.604$, $p=0.003$ ) and the entire genus ( $\operatorname{res}_{\text {clade } 1}=0.723$ vs. res $_{\text {genus }}=0.664, p=0.010$ ). The correlations between the incongruence and resolution metrics were $\rho=0.405$ and $\rho=0.484$ for clade 1 and 2 , respectively.

Figure 4 shows the difference in comparison metrics and average bootstrap support (boot) when markers were randomly concatenated and an optimised combination of markers was selected. Table 4 lists optimal sets of two to seven markers for use in studies of the Francisella genus. Summary statistics of the optimal combinations of markers in the entire genus are summarised in Additional file 5. Results of the optimisation analyses of the separate clades are not shown. Compared to random concatenation of sequence markers, the Francisella genus topology from an optimised set of markers reduced the difference in resolution by on average 50 - 59\% and totally eliminated incongruences. The suggested combination of five gene fragments in [34] resulted in a topology comparison with res $=0.471$ and inc $=0.217$, whereas the corresponding optimal topology resulted in res $=0.176$ and inc $=0.000$. The average bootstrap support of the optimised topologies compared to the average bootstrap of random marker topologies was significantly higher for congruence at the 5 marker level (boot $_{\text {opt }}=88.33$ vs. boot $_{\text {rand }}=86.38, p<0.001$ ), 6 mar-

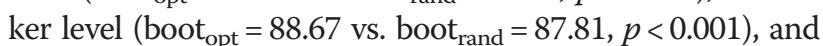
7 marker level $\left(\right.$ boot $_{\mathrm{opt}}=88.92$ vs. boot $_{\mathrm{rand}}=88.29$, $p<0.001$ ), as well as for resolution at the 6 marker level (boot $_{\mathrm{opt}}=90.71 \mathrm{vs.}$ boot $\left._{\mathrm{rand}}=87.81, p<0.001\right)$.

\section{Discussion}

Knowledge about theoretical limitations of marker assays is important for the successful detection and identification of bacteria in research as well as public health contexts. Existing methods for detection and identification 

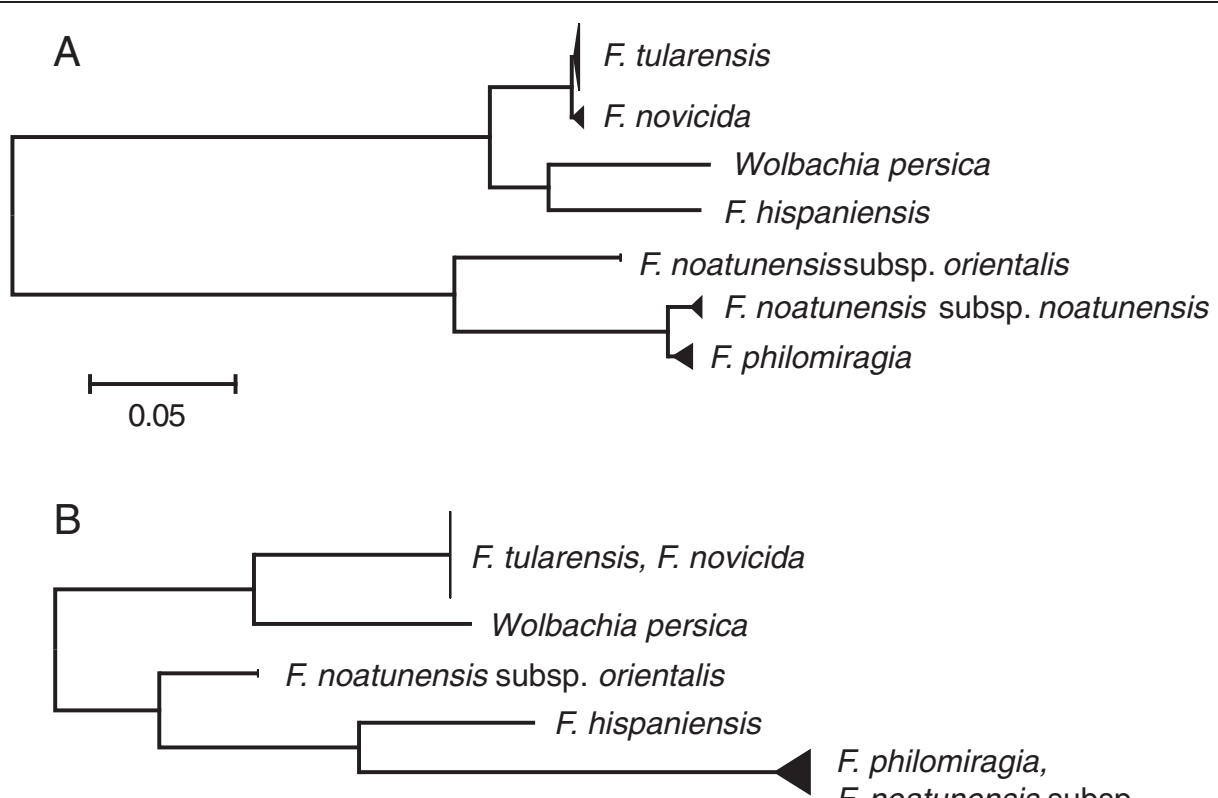

F. philomiragia, F. noatunensis subsp.

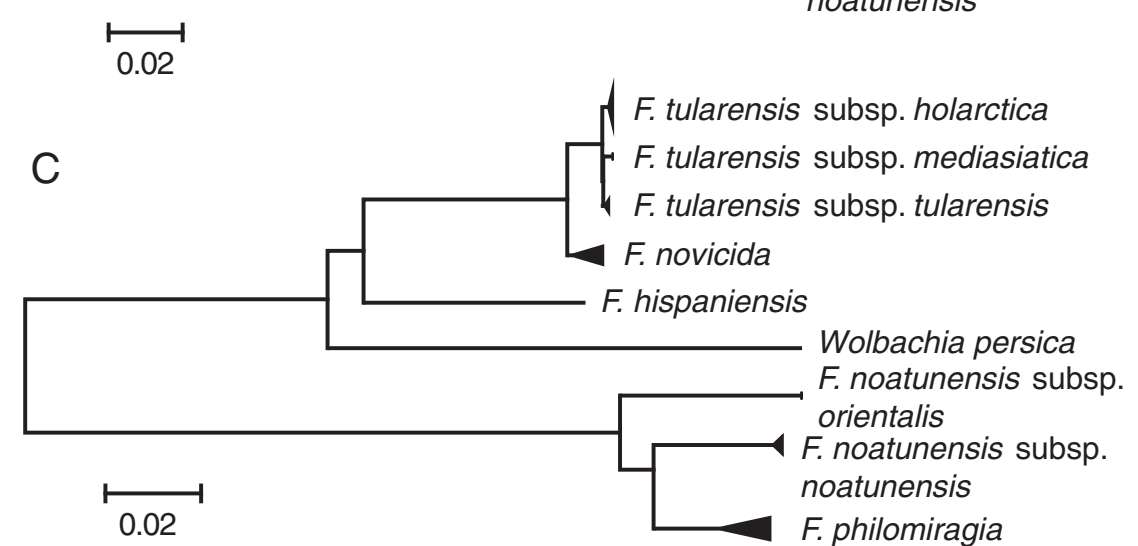

Figure 3 Single-marker phylogenies. Single-marker phylogeny of the Francisella genus: (A) highest ranked marker 08-fabH, (B) lowest ranked marker 33-rpoB, and (C) whole-genome phylogeny. Rank is based on difference in resolution between alternative and whole-genome topology.

of Francisella were developed with limited knowledge about the genetic diversity within the Francisella genus. From a clinical perspective, the lack of knowledge of diversity in the environment may be of minor importance since diagnostic sampling is performed on humans or animals suspected of having the disease. In contrast, use of the same detection assays for environmental sampling can lead to problems with false positive results. The recent increase in publicly available genome sequences enables development of improved detection and identification methods for both purposes. The emergence of high-throughput typing of large collections of bacterial strains targeting single amplicons is likely to mean that the targeting of single-marker regions will continue to be important in the future [20].

In this study, we evaluated 38 published markers (Table 2) against the current known diversity of the
Francisella genus. It is important to note that the studies from which the markers were gathered differed widely in scope. Some studies were designed to only cover a specific species and exclude others, whereas in other studies it was not of interest or even possible to study all the Francisella species included here. Several of the included markers were amplifying sequence products for species not included in previous studies of Francisella, e.g. F. hispaniensis, F. noatunensis and W. persica. As many as one third of the markers amplified all the included subspecies and approximately half of the markers amplified products for $F$. hispaniensis and/or W. persica together with clade 1 or clade 2 . This indicates that strains belonging to $F$. hispaniensis, $W$. persica, $F$. noatunensis are responsible for several false identifications. It should be pointed out that we have only considered sequence based markers here. Other type of markers and marker 

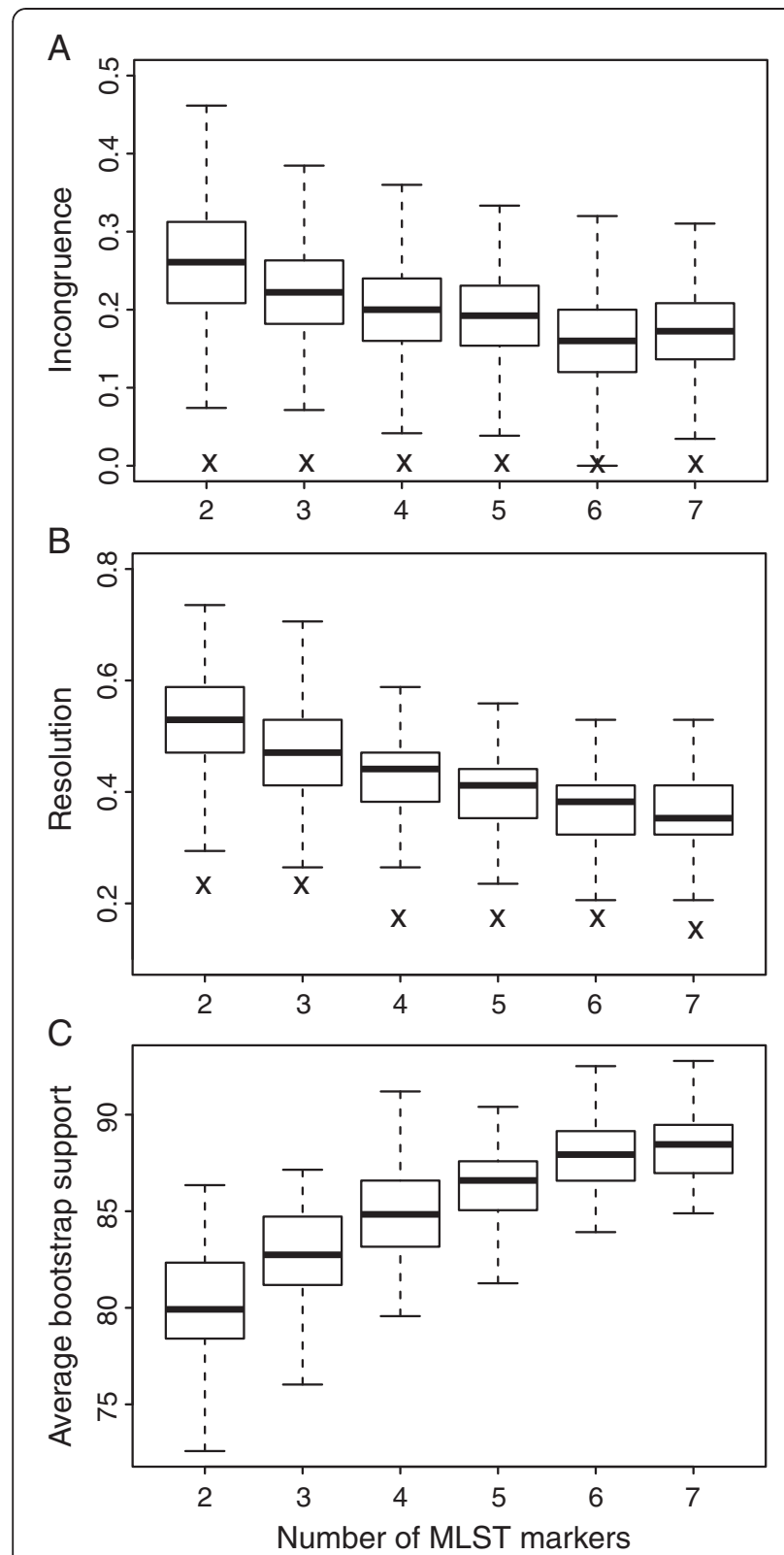

Figure 4 The impact of the number of markers on phylogenetic parameters. The effect of concatenating sequence markers on topology (of the Francisella genus) in comparison with the wholegenome tree for (A) incongruence score, (B) resolution score, and (C) average bootstrap support from 1000 replicates. The results of the optimised topology comparisons are shown as crosses.

combinations can be fruitful, in particular for construction of sub-species specific assays, which has been shown by e.g. combining variable-number of tandem repeats (VNTR) and insertion-deletion (indel) markers [35] or SNP and indel markers [36].

Specificity is especially important for markers designed for detection. The results of the investigated detection markers suggested that the specificity was questionable for the majority of them. The marker 22-lpnA [37,38], designated for $F$. tularensis detection, was found to also amplify $F$. hispaniensis FSC454 [39]. In the present study, the primers of the genus-specific marker 13-fopA [16] were not predicted to amplify any of the included $F$. philomiragia, whereas in the original publication they were reported to amplify all included F. philomiragia isolates. Probably a large unknown diversity exists within this species. For almost all 11 detection markers for Francisella tularensis, there was a significant risk of false-negative results caused by unwanted mismatches for isolates that should be detected. In conclusion, primer sequences need to be continually evaluated and redesigned using up-to date knowledge of the genetic diversity of the targeted sequences to minimise the likelihood of false-positive or -negative results. A similar conclusion was published by [40] where false-positive and -negative hits of primers against publically available sequences in various species of bacteria were evaluated with the result of high degree of primer mismatch in Haemophilus influenza, Pseudomonas aeruginosa and Escherichia coli. Hence, primer miss-match seems to be a general problem within prokaryotes. Our evaluation approach for primers could subsequently be of benefit to the microbiological community.

In order to compare analyses based on PCR-based sequence data with analyses based on whole-genome data for making phylogenetic inferences, we partitioned the popular RF metric into two separate metrics, incompatibility and resolution, to enable comparison of an alternative topology with a reference topology. These two metrics explain different characteristics, which allow a particular question to be considered when evaluating the phylogeny of bacteria given the reference topology. In the genomes of Francisella analysed here, these two metrics were correlated and therefore displaying similar metric characteristics, albeit with some exceptions, particularly in the clade 1 analysis. The incompatibility metric was negatively correlated with nucleotide diversity, whereas the opposite was found for the resolution metric, which highlights differences in the characteristics of these metrics. This finding suggests that singlenucleotide polymorphisms (SNPs) in marker-sequence regions increase the resolution but may also compromise the phylogenetic signal. One possible explanation for the incompatibility of SNPs and whole-genome phylogeny is the presence of recombination within sequence fragments, which has been suggested by several previous analyses of pathogenic bacteria populations; i.e. Neisseria meningitidis [22,25,41], Staphylococcus aureus [22,42] and Escherichia coli $[22,43]$. Nonetheless, for analysis of large numbers of bacterial strains showing conflicting topologies using different combinations of markers, our proposed comparison metrics are useful measures. To 
Table 4 Summary of the optimisation procedure for resolution (res) and congruence (inc) in the Francisella genus where the consensus set of markers are highlighted according to how often they are selected in the optimal partitions of markers; position 1 corresponds to the most represented marker

\begin{tabular}{|c|c|c|c|c|c|c|c|c|}
\hline No of markers & $\begin{array}{l}\text { Position } \\
\text { Metric }\end{array}$ & 1 & 2 & 3 & 4 & 5 & 6 & 7 \\
\hline \multirow[t]{2}{*}{2} & res & 08-fabH & 35-tpiA & & & & & \\
\hline & inc & 08-fabH & 35-tpiA & & & & & \\
\hline \multirow[t]{2}{*}{3} & res & 08-fabH & 35-tpiA & 24-IpnB & & & & \\
\hline & inc & 08-fabH & 35-tpiA & $02-16 \mathrm{~s}$ & & & & \\
\hline \multirow[t]{2}{*}{4} & res & 08-fabH & 35-tpiA & 24-IpnB & 27-parc & & & \\
\hline & inc & 35-tpiA & 08-fabH & $01-165$ & $02-16 s$ & & & \\
\hline \multirow[t]{2}{*}{5} & res & 08-fabH & 35-tpiA & 24-IpnB & 27-parc & 22-IpnA & & \\
\hline & inc & 35-tpiA & 08-fabH & 24-IpnB & $27-\operatorname{parc}$ & 33-rрoB & & \\
\hline \multirow[t]{2}{*}{6} & res & 08-fabH & 24-IpnB & 35-tpiA & 27-parC & 22-IpnA & 25-mdh & \\
\hline & inc & 35-tpiA & 08-fabH & 24-IpnB & $04-16 s$ & $01-165$ & 33-rрoB & \\
\hline \multirow[t]{2}{*}{7} & res & 08-fabH & 35-tpiA & 24-IpnB & 26-muts & 27-parC & 18-groEL & 22-IpnA \\
\hline & inc & 35-tpiA & 08-fabH & $01-165$ & $04-16 s$ & 24-IpnB & 27-parc & 25 -mdh \\
\hline
\end{tabular}

Markers $02-16 \mathrm{~s}+\mathrm{ItS}+23 \mathrm{~s}$ and $04-16 \mathrm{~s}+\mathrm{It} \mathrm{t}+23 \mathrm{~s}$ are abbreviated as $02-16 \mathrm{~s}$ and $04-16 \mathrm{~s}$, respectively.

determine whether the observed topological differences could have occurred by chance, our comparison approach can be combined with a statistical test, such as the $\mathrm{SH}$ test applied here or an alternative test, e.g. $[44,45]$.

Most incompatibilities were associated with the topologies that included all strains, whereas the level of incompatibility was significantly lower for clade 1 , with topologies being totally compatible in many cases. These results indicate that the clonal frame is maintained within the F. tularensis clade, but it is disrupted at the genus level and in clade 2. Most incompatibilities were a result of $F$. philomiragia, $F$. novicida, W. persica and $F$. hispaniensis strains that were misplaced in the singlemarker cases, which suggests that recombination is the main evolutionary force that promotes incongruences in Francisella, as pointed out by, e.g. $[7,18]$. The reduction of recombination rate in clade 1 might, in turn, reflect barriers to gene flow between ecological and geographical clusters among sub-species [7,46-49].

Our result suggests that no single-marker topology of the entire genus is able to assign all strains to the subspecies defined by the whole genome topology. In fact, some marker topologies, such as $02-16 \mathrm{~s}+\mathrm{ItS}+23 \mathrm{~s}$ and 24-rpoB, made deviating assignments in more than $70 \%$ of the cases. The reason for the low success rate of assigned strains to their corresponding sub-species is mainly poor resolution, which meant that typically all $F$. tularensis strains displayed identical sequences. Most topologies assigned all strains to the same main clades as in the whole genome phylogeny, with a few exceptions: 33-rpoB assigned F. hispaniensis to clade 2 and 19-iglC assigned W. persica to clade 2, in subgroup $F$. noatunensis subsp. orientalis (in both assignments). This is an interesting observation as rpoB was recently suggested as an alternative marker to $16 \mathrm{~S}$ rDNA in metagenomic studies [21].

The level of incompatibility and difference in resolution compared to the whole-genome reference topology were decreased, in some cases by a considerable amount, by selecting an optimal combination of markers. Moreover, topologies based on an optimal set of markers significantly increased the average statistical support (i.e. average bootstrap). Generally, both the degree of compatibility and resolution were improved by concatenating sets of two to seven markers in all possible combinations. However, some combinations, in particular considering incompatibility, might result in poorer topologies than for an estimated topology based on a single marker. This observation is consistent with previous work where concatenation of sequence data have resulted in biased phylogenetic estimates [50]. All incompatible phylogenetic signals were removed in topologies based on optimised sets of two to seven markers, in contrast to random concatenation. Totally congruent topologies were obtained by concatenating as few as only two markers $(08-\mathrm{fabH}$ and 35-tpiA). These two markers were included in all optimal sets. Hence, by selecting an optimal set of markers, a large improvement in resolution and compatibility can be obtained over random concatenation.

An exhaustive search strategy was employed to find the optimal set of markers since the total number of available markers was relatively small. It should be pointed out that the number of possible marker combinations increases rapidly with the number of markers considered and soon becomes computationally intractable. As all the 
742 gene fragments of the core genome in the analysed population have recently become available in [3], an interesting extension to the current work would be to find the optimal set of markers based on all those genes. Such an optimisation could be carried out by utilising one of the myriad of available optimisation techniques, such as a simulated annealing approach $[51,52]$. It should be noted that we do only try to minimize the value of the objective metrics, incongruence or resolution difference, with respect to the whole-genome topology. There is no guarantee that the whole genome topology accurately resembles the true underlying species topology as systematic errors and statistical inconsistencies in the phylogenetic inference method could be amplified when analyzing whole genome data [50,53-55].

By demonstrating the potential of establishing robust bacterial phylogenies using sample sequencing of only a few markers, we believe that the framework presented here could serve as a foundation for population analyses as well as for identifying and attributing unknown pathogenic strains to the correct subspecies.

\section{Conclusions}

The results of this study suggest that several of the investigated markers designed to be diagnostic exhibit a considerable level of unspecificity. Hence, several of the currently used primers need to be redesigned to avoid false-positive results. This arises because of a previous lack of knowledge about genetic diversity within the Francisella genus represented by, e.g. strains belonging to F. hispaniensis and among FLEs. By employing sample sequencing of DNA markers to make phylogenetic inferences, we revealed incompatibilities among topologies that included all considered Francisella strains but not among topologies that included only clade 1 strains containing F. tularensis. An estimated topology based on optimised combination of markers drastically reduced incompatibility and resolution differences compared to topologies obtained by random concatenation and at the same time improved the average bootstrap support, using the whole genome phylogeny as a reference. Implementation of such an optimisation framework based on accurate reference topology would help to improve assays for detection and identification purposes, which are of considerable importance in a number of research fields, such as for improving biosurveillance systems and inferring evolutionary histories.

\section{Methods}

\section{Bacterial strains}

A total of 37 genome sequences (Table 1) were selected to represent the known diversity of Francisella. This collection included both pathogenic and non-pathogenic strains and could be divided into two major clades. The public-health perspective was represented by 22 strains of the human pathogen F. tularensis (clade 1) and the fish-farming industry and health perspective was represented by 13 strains of $F$. noatunensis and F. philomiragia, which are all fish pathogens (clade 2). In addition, the strain Wolbachia persica FSC845, representing the FLEs, and the newly discovered F. hispaniensis FSC454 were included. More detailed information about the included strains has been published elsewhere [3].

\section{PCR markers}

The study focused on a set of 38 markers used in detection or identification of Francisella (Table 2). A subset of 13 markers (01-16S [14,37,38,56], 22-lpnA [19,37,38,56,57], 13-fopA, 19-iglC, 21-ISFtu2, 23-lpnA [9,16], 11-fopA-in, 12fopA-out [15], 14-FtM19 [56,58], 16-FTT0376, 17-FTT0523 [17], 20-ISFtu2 [56,59] and 28-pdpD [56,60]) were originally designed primarily for real-time PCR molecular detection of Francisella at different taxonomic levels; genus, species or subspecies (here called detection markers).

A subset of 25 markers $(02-16 \mathrm{~S}+\mathrm{ItS}+23 \mathrm{~S}, 03-16 \mathrm{~S}+$ ItS + 23S， 04-16S + ItS + 23S， 10-fopA， 18-groEL，24lpnB, 33-rpoB, 34-sdhA [34], 05-aroA, 06-atpA, 27-parC, 29-pgm, 36-tpiA, 37-trpE, 38-uup [18,61] 07-dnaA, 09fopA, 26-mutS, 30-prfB, 31-putA, 35-tpiA [19], 08-fabH [62], 25-mdh [63,64] 32-rpoA [64], 15-FtM19 [65]), which were originally designed for PCR-based identification (here called identification markers), were also included.

The primer specificity was tested for all 38 markers. In the topological comparisons and optimisation procedures, 28, 27 and 26 markers were used for clade 1, clade 2 and the whole-genome data, respectively (see Additional file 1 for details).

\section{In silico PCR}

PCR fragments were assumed to result from all included genomes rather than exclusively the genomes considered in developing the marker. An in silico PCR fragment was first generated for one selected isolate (F. tularensis subsp. tularensis SCHU S4, F. tularensis subsp. holarctica FSC200 or F. noatunensis subsp. noatunensis FSC769) using multithreaded electronic PCR (mismatches allowed $=4$, expected length $=2000 \mathrm{bp}$, margin $=400 \mathrm{bp}$, honouring IUPAC ambiguity in STS) [66], which is an enhanced version of electronic PCR [67]. This fragment was then aligned to the rest of the genomes using Exonerate v2.2.0 (model: est2genome, percent threshold $=70$, score threshold $=50$, maxintron length $=2500$ ) [68]. Finally, all fragments for each marker were aligned using MUSCLE v3.7 using default settings [69].

\section{PCR-primer scoring}

Primer specificity was evaluated by scoring each primer sequence against the corresponding in silico generated target sequences using PrimerProspector [70]. To direct 
the scoring to the region where the primer sequence aligned for all strains, the primer region was extracted from the alignment and used alone as input to the scoring software. The weighted score was calculated based on 3' mismatch (penalty 1 per mismatch, 3' length 5), non-3' mismatch (penalty 0.4 per mismatch), last-base mismatch (penalty 3 per mismatch), non 3' gap (penalty 1 per gap) and 3' gap (penalty 3 per gap). The lowest possible score in this type of calculation is zero, which is only achieved when the primer is a perfect match. The score, which is based on mismatches and gaps, is dependent on primer length, and thus a max score cannot be given. The limit for a possible PCR amplification was set to 2, in agreement with the NCBI PrimerBLAST default primer specificity stringency setting for amplification, i.e. at least two mismatches in the 3' region. According to latter system, scores below two are regarded as low scores, whereas scores greater than or equal to two are regarded as high scores. Calculated scores for forward and reverse primers for each strain were clustered with DIvisive ANAlysis clustering in the cluster package [71] and then plotted in a heatmap using the ggplot2 package [72] in R v2.13.1 [73].

\section{Phylogenetic analysis}

Phylogenetic trees were inferred using two alternative methods: neighbour joining (NJ) [74] and maximum likelihood (ML) [75]. The software packages PhylML 3.0 [76,77] and Phylip [78] were used. In the NJ analysis, 1000 bootstrap replicates were calculated in the software Seqboot and summarised in the Consense software. The genetic distances between strains were estimated with the software Dnadist by employing the F84 nucleotide substitution model [79]. The NJ tree was inferred with the Neighbour software, in the Phylip package [76]. By using the software jModelTest [80], we were able to evaluate alternative nucleotide substitution models for the maximum likelihood analysis and perform model averaging [81], in which the alternative models were weighted based on the fit to the data and model complexity (i.e. the number of effective parameters in each substitution model) using the Bayesian information criterion (BIC) [82]. Substitution models with unequal base frequencies, a proportion of invariable sites, $\alpha$, and allowance for rate variation among sites, $\Gamma$, were included. The number of discrete gamma categories was 4 . In total, we considered 24 alternative substitution models in the model-averaging process. The more computationally intense ML procedure was chosen to estimate phylogenies in the single-marker analysis, whereas the rapid NJ method was utilised in the multiple marker analyses. The wholegenome phylogeny was estimated with both the ML and NJ methods by considering 20,072 SNPs on the core genome of all 37 genomes. The SNPs were obtained using the same procedure as in [3], where the Mauve software [83] with default options was used to perform multiple genome alignment and in-house perl-script was used to identify the SNPs based on the obtained alignments. As both ML and NJ methods resulted in virtually identical phylogenies, we concluded that the choice of estimation method did not have a significant impact on the evaluation of the sequence-marker topologies.

\section{Phylogenetic-topology comparison}

To check for and quantify the degree of compatibility between the phylogenetic trees estimated with markersequence data and the whole-genome tree (i.e. two trees with nested taxa), bipartitions in the marker tree were checked for their presence/absence in the whole-genome tree. In trees with missing sequences, the corresponding leaves were removed from the whole-genome tree using the $R$ package ape [84]. The output, i.e. number of absent bipartitions, were normalised by the total number of bipartitions in the marker tree. This topology metric was denoted inc throughout the study. For perfectly compatible trees, no bipartitions in the marker tree should be absent in the whole-genome tree. To obtain the bipartitions at the internal edges of the trees, the output from the Consense software in the Phylip package [78], together with an in-house Perl script (available upon request), were used. The inc metric is similar to the RF distance [26], although the RF metric counts the number of bipartitions not present in the other tree for both trees. Therefore, the RF metric measures both the degree of incongruence and the difference in resolution between reference and alternative topologies. By modifying the RF distance metric, the degree of incongruence can be quantified more precisely and also separated from the difference in resolution between the compared topologies. In a similar manner, a Perl script was implemented to count the number of bipartitions present in the wholegenome topology that were absent in the alternative topology (i.e. difference in resolution, denoted res) and to normalise the output to vary between 0 and 1 . As a reference, RF distances (also known as symmetric differences) implemented in the Treedist software [78] were used. To investigate the success of the marker tree to allocate a strain to its corresponding sub-species family (according to the whole genome phylogeny), bipartition scoring in the Consense software was used and the output was compared to the pre-defined subspecies bipartitions according to the whole-genome tree. In addition, we investigated whether strains were assigned to the corresponding main clades of the entire Francisella genus, reporting the proportion of misidentified strains on each clade. Finally, we considered the average bootstrap support of each marker tree. 
It is important to consider a statistical test for topological incongruence as stochastic effects in the evolution of the sequences results in incongruence between the compared trees. To address this issue, we employed the Shimodaira-Hasegawa $(\mathrm{SH})$ test [85], which is a non-parametric test for determining whether there are significant differences between conflicting topologies in specific sequence data. The null hypothesis of the $\mathrm{SH}$ test assumed that the compared topologies were equally probable given the data. Here, we tested the marker topologies and the wholegenome topology on each respective marker sequence using the phyML software package by fixing the topologies and optimising the substitution model and branch-length parameters. The $\mathrm{SH}$ test was performed within the CONSEL software package [86], which takes the output from phyML as input. Since multifurcations in topologies are strongly penalised in the phyML software, we resolved the topologies into bifurcating trees using the $\mathrm{R}$ package ape [84]. The substitution model selected in the phyML analysis was based on the preferred substitution model of the jModelTest analysis. To test whether clades differed in incongruence or resolution, a Wilcoxon rank sum test with continuity correction was utilised, implemented in the R statistical package [73]. We used Spearman's rank correlation coefficient, $\rho$, to quantify correlations between metrics and the average pairwise nucleotide diversity, $\pi$, of the clades.

\section{Optimisation procedure}

Since the number of included sequence markers in this study was moderate, we searched through all possible combinations of markers (i.e. an exhaustive search). We performed two separate analyses, one for each of the metrics used: incongruence and difference in resolution between topologies. The marker configuration(s) resulting in the lowest metric value were saved. The code was written in Perl and is available upon request from JA.

To test whether the average bootstrap support obtained from optimised topologies and topologies generated by random concatenation differed, we again made use of the Wilcoxon rank sum test with continuity correction in cases where more than 10 optima were found. The null hypothesis was that the level of average bootstrap support was equivalent for the optimised and randomised topologies. Due to the high computational demands, we only analysed 100 topologies obtained by random concatenation of sequences with respect to bootstrap support. Furthermore, we compared the optimal topology identified here to the topology obtained by analysing the sequence combination suggested by [34]: 33-rpoB, 10-fopA, 18-groEL, 24-lpnB and 34-sdhA.

\section{Additional files}

Additional file 1: Summary of earlier published and current results of investigated sequence markers. A list of earlier published as well as current results of the specificity of each marker at subspecies level, presence/absence of the markers in the different clades, details of which parts of the study the marker was included and marker type.

Additional file 2: Single-marker topologies. A zip-file containing all single-marker topologies in pdf format obtained from the modelaveraging phylogenetic analysis using jModelTest.

Additional file 3: Parameter estimates obtained from the phylogenetic analysis. Summary statistics of the single-marker phylogenetic analysis. The most optimal DNA substitution model was selected by BIC implemented in jModelTest. Standard errors of average bootstrap supports are shown in parentheses. The estimated proportion of invariable sites is the expected frequency of sites that do not evolve.

Additional file 4: Table of single-marker results. Comparison of inferred single-gene topologies to the whole-genome topology with respect to RF distance degree of incongruence, difference in resolution, the proportion of misidentified strains and SH test of incongruence. To test alternative topologies for markers with missing sequences, the corresponding leaves were removed from the whole-genome tree.

Additional file 5: Optimal set of marker partitions. Optimisation of the subset of two to seven marker-sequence topologies to minimise incongruences and difference in resolution compared to the wholegenome topology. The numbers show the percentage of each marker included in the optimal configurations. The proportion of strains misplaced in the tree, average bootstrap support of optimal topologies and the $\mathrm{SH}$ test of incongruence is also reported. The total number of global optima was calculated from the output of the heuristic search analyses.

\section{Abbreviations}

BIC: Bayesian information criterion; Clade 1: Population including F. tularensis subspecies; Clade 2: Population including F. noatunensis subspecies and F. philomiragia; Entire genus: Entire genus all included strains representing all known subspecies; FLEs: Francisella like endosymbionts; GTR: Generalised time reversible; HKY85: Hasegawa-Kishino-Yano; Indel: Insertion-deletion; JC: Jukes Cantor; ML: Maximum likelihood; NGS: Next generation sequencing; NJ: Neighbour joining; RF: Robinson-Foulds; SH: Shimodaira-Hasegawa; SNP: Single-nucleotide polymorphisms; VNTR: Variable number of tandem repeats.

\section{Authors' contributions}

JA and CÖ wrote script code, extracted and analysed the data; JA, CÖ, and AS wrote the manuscript; KS, PLI, AJ, MF, PLA contributed to writing the manuscript; AJ, MF, PLA and AS organised and conceived the study. All authors read and approved the final manuscript.

\section{Acknowledgements}

This project was funded by the Swedish Ministry of Foreign Affairs, project A4952, the Swedish Civil Contingencies Agency, project B4055 and the Swedish Ministry of Defence, project A404012. We wish to thank the associate editor and three anonymous reviewers for comments that improved an earlier version of the paper.

\section{Author details}

${ }^{1}$ Division of CBRN Security and Defence, FOI, Swedish Defence Research Agency, SE- 906 21, Umeå, Sweden. '2Department of Clinical Microbiology, Umeå University, SE-901 85, Umeå, Sweden. ${ }^{3}$ Laboratory for Molecular Infection Medicine Sweden (MIMS), Umeå University, SE-901 87, Umeå, Sweden.

Received: 4 June 2012 Accepted: 31 August 2012

Published: 25 September 2012

\section{References}

1. Rotz LD, Khan AS, Lillibridge SR, Ostroff SM, Hughes JM: Public health assessment of potential biological terrorism agents. Emerg Infect Dis 2002, 8:225-230. 
2. Beran GW, Steele JH: Handbook of Zoonoses: Section A: Bacterial, Rickettsial, Chlamydial, and Mycotic Zoonoses. 2nd edition. Boca Raton: CRC-Press; 1994.

3. Sjödin A, Svensson K, Öhrman C, Ahlinder J, Lindgren P, Duodu S, Johansson A, Colquhoun DJ, Larsson P, Forsman M: Genome characterisation of the genus Francisella reveals similar paths of host adaption in pathogens of mammals and fish. BMC Genomics 2012, 13:268.

4. Hollis DG, Weaver RE, Steigerwalt AG, Wenger JD, Moss CW, Brenner DJ: Francisella philomiragia comb. nov. (formerly Yersinia philomiragia) and Francisella tularensis biogroup novicida (formerly Francisella novicida) associated with human disease. J Clin Microbiol 1989, 27:1601-1608.

5. Johansson A, Celli J, Conlan W, Elkins KL, Forsman M, Keim PS, Larsson P, Manoil C, Nano FE, Petersen JM, Sjöstedt A: Objections to the transfer of Francisella novicida to the subspecies rank of Francisella tularensis. Int $J$ Syst Evol Microbiol 2010, 60:1717-1718. author reply 1718-20.

6. Busse H-J, Huber B, Anda P, Escudero R, Scholz HC, Seibold E, Splettstoesser WD, Kämpfer P: Objections to the transfer of Francisella novicida to the subspecies rank of Francisella tularensis - response to Johansson et al. Int J Syst Evol Microbiol 2010, 60:1718-1720.

7. Larsson $P$, Elfsmark $D$, Svensson $K$, Wikström $P$, Forsman $M$, Brettin T, Keim P, Johansson A: Molecular evolutionary consequences of niche restriction in Francisella tularensis, a facultative intracellular pathogen. PLOS Path 2009, 5:e1000472.

8. Johansson A, Ibrahim A, Göransson I, Eriksson U, Gurycova D, Clarridge JE, Sjöstedt A: Evaluation of PCR-based methods for discrimination of Francisella species and subspecies and development of a specific PCR that distinguishes the two major subspecies of Francisella tularensis. J Clin Microbiol 2000, 38:4180-4185.

9. Barns SM, Grow CC, Okinaka RT, Keim P, Kuske CR: Detection of diverse New Francisella-like bacteria in environmental samples. Appl Environ Microbiol 2005, 71:5494-5500.

10. Keim P, Pearson T, Okinaka R: Microbial forensics: DNA fingerprinting of Bacillus anthracis (Anthrax). Anal Chem 2008, 80:4791-4800.

11. Shea DA, Lister SA: The BioWatch Program: Detection of Bioterrorism, Congressional Research Service.Report No. RL 32152. Washington, DC: Library of Congress; 2012. November 19, 2003. Accessed online at http://www.fas. org/sgp/crs/terror/RL32152.html on March 9, 2012

12. Kman NE, Bachmann DJ: Biosurveillance: a review and update. Adv Prev Med 2012, 2012:301408.

13. Bush NS: BioWatch: case for change of traditional leadership to improve performance. Monterey: Master's Thesis. Naval Postgraduate School; 2009.

14. Forsman M, Sandström G, Sjöstedt A: Analysis of $16 \mathrm{~S}$ ribosomal DNA sequences of Francisella strains and utilization for determination of the phylogeny of the genus and for identification of strains by PCR. Int J Syst Bact 1994, 44:38-46.

15. Higgins JA, Hubalek Z, Halouzka J, Elkins KL, Sjostedt A, Shipley M, Ibrahim MS: Detection of Francisella tularensis in infected mammals and vectors using a probe-based polymerase chain reaction. Am J Trop Med Hyg 2000, 62:310-318

16. Versage JL, Severin DDM, Chu MC, Petersen JM: Development of a multitarget real-time TaqMan PCR assay for enhanced detection of Francisella tularensis in complex specimens. J Clin Microbiol 2003, 41:5492-5499.

17. Mitchell JL, Chatwell N, Christensen D, Diaper H, Minogue TD, Parsons TM, Walker B, Weller SA: Development of real-time PCR assays for the specific detection of Francisella tularensis ssp. tularensis, holarctica and mediaasiatica. Mol Cell Probe 2010, 24:72-76.

18. Svensson $K$, Larsson $P$, Johansson D, Byström M, Forsman M, Johansson A: Evolution of subspecies of Francisella tularensis. J Bact 2005, 187:3903-3908.

19. Nübel $U$, Reissbrodt $R$, Weller $A$, Grunow $R$, Porsch-Ozcürümez $M$, Tomaso $H$, Hofer E, Splettstoesser W, Finke E-J, Tschäpe H, Witte W: Population structure of Francisella tularensis. J Bact 2006, 188:5319-5324.

20. Singh P, Foley SL, Nayak R, Kwon YM: Multilocus sequence typing of Salmonella strains by high-throughput sequencing of selectively amplified target genes. J Microbiol Meth 2012, 88:127-133.

21. Vos M, Quince C, Pijl AS, de Hollander M, Kowalchuk GA: A comparison of rpoB and 16S rRNA as markers in pyrosequencing studies of bacterial diversity. PLoS One 2012, 7:e30600.

22. Feil EJ, Holmes EC, Bessen DE, Chan MS, Day NP, Enright MC, Goldstein R, Hood DW, Kalia A, Moore CE, Zhou J, Spratt BG: Recombination within natural populations of pathogenic bacteria: short-term empirical estimates and long-term phylogenetic consequences. P Natl Acad Sci USA 2001, 98:182-187.

23. Lerat $E$, Daubin $V$, Moran NA: From gene trees to organismal phylogeny in prokaryotes: the case of the gamma-Proteobacteria. PLOS Biol 2003, 1:E19.

24. Noël C, Dufernez F, Gerbod D, Edgcomb VP, Delgado-Viscogliosi P, Ho L-C, Singh M, Wintjens R, Sogin ML, Capron M, Pierce R, Zenner L, Viscogliosi E: Molecular phylogenies of blastocystis isolates from different hosts: implications for genetic diversity, identification of species, and zoonosis. J Clin Microbiol 2005, 43:348-355.

25. Holmes EC, Urwin R, Maiden MC: The influence of recombination on the population structure and evolution of the human pathogen Neisseria meningitidis. Mol Biol Evol 1999, 16:741-749.

26. Robinson D, Foulds L: Comparison of phylogenetic trees. Math Biosci 1981, 53:131-147.

27. Kuhner MK, Felsenstein J: A simulation comparison of phylogeny algorithms under equal and unequal evolutionary rates. Mol Biol Evol 1994, 11:459-468.

28. Steel MA, Penny D: Distributions of tree comparison metrics-some new results. Syst Biol 1993, 42:126-141.

29. Waterman MS, Smith TF: On the similarity of dendrograms. J Theor Biol 1978, 73:789-800

30. Primer-BLAST, NCBI. http://www.ncbi.nlm.nih.gov/tools/primer-blast.

31. Tavare S: Some probabilistic and statistical problems in the analysis of DNA sequences. Lect Math Life Sci 1986, 17:57-86.

32. Hasegawa M, Kishino $H$, Yano $T$ : Dating of the human-ape splitting by a molecular clock of mitochondrial DNA. J Mol Evol 1985, 22:160-174.

33. Jukes TH, Cantor CR: Evolution of protein molecules. In Mammalian Protein Metabolism vol.3. Edited by Munro HN. New York: Academic Press; 1969:21-132.

34. Bohle H, Tapia E, Martínez A, Rozas M, Figueroa A, Bustos P: Francisella philomiragia, bacteria asociada con altas mortalidades en salmones del Atlántico (Salmo salar) cultivados en balsas-jaulas en el lago Llanquihue. Arch Medi Veter 2009, 41:237-244

35. Larsson P, Svensson K, Karlsson L, Guala D, Granberg M, Forsman M Johansson A: Canonical insertion-deletion markers for rapid DNA typing of Francisella tularensis. Emerg Infect Diseases 2007, 13:1725-1732.

36. Svensson K, Granberg M, Karlsson L, Neubauerova V, Forsman M, Johansson A: A real-time PCR array for hierarchical identification of Francisella isolates. PLoS One 2009, 4:e8360.

37. Sjöstedt A, Eriksson U, Berglund L, Tärnvik A: Detection of Francisella tularensis in ulcers of patients with tularemia by PCR. J Clin Microbiol 1997, 35:1045-1048

38. Johansson A, Berglund L, Eriksson U, Göransson I, Wollin R, Forsman M Tärnvik A, Sjöstedt A: Comparative analysis of PCR versus culture for diagnosis of ulceroglandular tularemia. J Clin Microbiol 2000, 38:22-26.

39. Versage JL, Severin DDM, Chu MC, Petersen JM: Development of a multitarget real-time TaqMan PCR assay for enhanced detection of Francisella tularensis in complex specimens. J Clin Microbiol 2003, 41:5492-5499.

40. Lemmon GH, Gardner SN: Predicting the sensitivity and specificity of published real-time PCR assays. Ann Clin Microbiol Antimicrob 2008, 7:18.

41. Urwin R, Holmes EC, Fox AJ, Derrick JP, Maiden MCJ: Phylogenetic evidence for frequent positive selection and recombination in the Meningococcal surface antigen PorB. Mol Biol Evol 2002, 19:1686-1694.

42. Sabat AJ, Wladyka B, Kosowska-Shick K, Grundmann H, van Dijl JM, Kowal J, Appelbaum PC, Dubin A, Hryniewicz W: Polymorphism, genetic exchange and intragenic recombination of the aureolysin gene among Staphylococcus aureus strains. BMC Microbiol 2008, 8:129.

43. Retchless AC, Lawrence JG: Phylogenetic incongruence arising from fragmented speciation in enteric bacteria. P Natl Acad Sci USA 2010, 107:11453-11458

44. Campbell V, Legendre P, Lapointe F-J: The performance of the Congruence Among Distance Matrices (CADM) test in phylogenetic analysis. BMC Evol Biol 2011, 11:64.

45. Irestedt M, Fjeldså J, Nylander JAA, Ericson PGP: Phylogenetic relationships of typical antbirds (Thamnophilidae) and test of incongruence based on Bayes factors. BMC Evol Biol 2004, 4:23.

46. Keim P, Johansson A, Wagner DM: Molecular epidemiology, evolution, and ecology of Francisella. Ann NY Acad Sci 2007, 1105:30-66.

47. Achtman M: Evolution, population structure, and phylogeography of genetically monomorphic bacterial pathogens. Ann Rev Microbiol 2008, 62:53-70. 
48. Keim PS, Wagner DM: Humans and evolutionary and ecological forces shaped the phylogeography of recently emerged diseases. Nat Rev Microbiol 2009, 7:813-821.

49. Thelaus J, Andersson A, Mathisen P, Forslund A-L, Noppa L, Forsman M: Influence of nutrient status and grazing pressure on the fate of Francisella tularensis in lake water. FEMS Microbiol Ecol 2009, 67:69-80.

50. Kubatko LS, Degnan JH: Inconsistency of phylogenetic estimates from concatenated data under coalescence. Syst Biol 2007, 56:17-24.

51. Spall JC: Introduction to Stochastic Search and Optimization. Hoboken, NJ: Wiley; 2003.

52. Kirkpatrick S, Gelatt CD, Vecchi MP: Optimization by simulated annealing Science 1983, 220:671-680.

53. Rodríguez-Ezpeleta N, Brinkmann H, Roure B, Lartillot N, Lang BF, Philippe H: Detecting and overcoming systematic errors in genome-scale phylogenies. Syst Biol 2007, 56:389-399.

54. Rannala B, Yang Z: Phylogenetic inference using whole genomes. Annu Rev Genomics Hum Genet 2008, 9:217-231.

55. Kumar S, Filipski AJ, Battistuzzi FU, Kosakovsky Pond SL, Tamura K: Statistics and truth in phylogenomics. Mol Biol Evol 2012, 29:457-472.

56. World Health Organization: WHO guidelines on tularaemia: epidemic and pandemic alert and response. Geneva: WHO; 2007:125

57. Berrada ZL, Telford SR: Diversity of Francisella species in environmenta samples from Martha's Vineyard, Massachusetts. M Microb Ecol 2010 59:277-283

58. Goethert HK, Shani I, Telford SR: Genotypic diversity of Francisella tularensis infecting Dermacentor variabilis ticks on Martha's Vineyard, Massachusetts. J Clin Microbiol 2004, 42:4968-4973.

59. Petersen JM, Schriefer ME, Carter LG, Zhou Y, Sealy T, Bawiec D, Yockey B, Urich S, Zeidner NS, Avashia S, Kool JL, Buck J, Lindley C, Celeda L, Monteneiri JA, Gage KL, Chu MC: Laboratory analysis of tularemia in wildtrapped, commercially traded prairie dogs, Texas, 2002. Emerg Infect Dis 2004, 10:419-425

60. Nano FE, Zhang N, Cowley SC, Klose KE, Cheung KKM, Roberts MJ, Ludu JS, Letendre GW, Meierovics Al, Stephens G, Elkins KL: A Francisella tularensis pathogenicity island required for intramacrophage growth. J Bact 2004, 186:6430-6436.

61. Birdsell DN, Stewart T, Vogler AJ, Lawaczeck E, Diggs A, Sylvester TL, Buchhagen $\mathrm{J}$, Auerbach RK, Keim P, Wagner DM: Francisella tularensis subsp. novicida isolated from a human in Arizona. BMC Res Note 2009, 2:223.

62. Rohmer L, Brittnacher M, Svensson K, Buckley D, Haugen E, Zhou Y, Chang J, Levy R, Hayden H, Forsman M, Olson M, Johansson A, Kaul R, Miller SI: Potential source of Francisella tularensis live vaccine strain attenuation determined by genome comparison. Infect Immun 2006, 74:6895-6906

63. Ottem KF, Nylund A, Karlsbakk E, Friis-Møller A, Krossøy B: Characterization of Francisella sp., GM2212, the first Francisella isolate from marine fish, Atlantic cod (Gadus morhua). Arch Microbiol 2007, 187:343-350.

64. Ottem KF, Nylund A, Karlsbakk E, Friis-Møller A, Kamaishi T: Elevation of Francisella philomiragia subsp. noatunensis Mikalsen et al. (2007) to Francisella noatunensis comb. nov. [syn. Francisella piscicida Ottem et al. (2008) syn. nov.] and characterization of Francisella noatunensis subsp. orientalis subsp. nov. J Appl Microbiol 2009, 106:1231-1243.

65. Johansson A, Farlow J Dukerich M, Chambers E, Byström M, Fox J Chu M, Forsman M, Sjöstedt A, Keim P: Worldwide genetic relationships among Francisella tularensis isolates determined by multiple-locus variablenumber tandem repeat analysis. J Bact 2004, 186:5808-5818.

66. Murphy K, Raj T, Winters RS: White PS: me-PCR: a refined ultrafast algorithm for identifying sequence-defined genomic elements. Bioinformatics 2004, 20:588-590

67. Schuler GD: Sequence mapping by electronic PCR. Genome Res 1997 7:541-550.

68. Slater GSC, Birney E: Automated generation of heuristics for biological sequence comparison. BMC Bioinf 2005, 6:31.

69. Edgar RC: MUSCLE: multiple sequence alignment with high accuracy and high throughput. Nucleic Acids Res 2004, 32:1792-1797.

70. Walters WA, Caporaso JG, Lauber CL, Berg-Lyons D, Fierer N, Knight R PrimerProspector: de novo design and taxonomic analysis of barcoded polymerase chain reaction primers. Bioinformatics 2011, 27:1159-1161.

71. Maechler M, Rousseeuw P, Struyf A, Hubert M, Hornik K: cluster: cluster analysis basics and extensions:; 2012
72. Wickham H: ggplot2: Eegant Graphics for Data Analysis (Use R!). New York: Springer; 2009

73. R Development Core Team: $R$ : a language and environment for statistical computing: 2011

74. Saitou N, Nei M: The neighbor-joining method: a new method for reconstructing phylogenetic trees. Mol Biol Evol 1987, 4:406-425.

75. Felsenstein J: Evolutionary trees from DNA sequences: a maximum likelihood approach. J Mol Evol 1981, 17:368-376.

76. Guindon S, Gascuel O: A simple, fast, and accurate algorithm to estimate large phylogenies by maximum likelihood. Syst Biol 2003, 52:696-704.

77. Guindon S, Dufayard J-F, Lefort V, Anisimova M, Hordijk W, Gascuel O: New algorithms and methods to estimate maximum-likelihood phylogenies: assessing the performance of PhyML 3.0. Syst Biol 2010, 59:307-321.

78. Felsenstein J: PHYLIP (Phylogeny Inference Package) version 3.5c. Distributed by the author. Seattle: Department of Genetics, University of Washington; 1993.

79. Felsenstein J, Churchill GA: A Hidden Markov Model approach to variation among sites in rate of evolution. Mol Biol Evol 1996, 13:93-104.

80. Posada D: jModelTest: phylogenetic model averaging. Mol Biol Evol 2008, 25:1253-1256

81. Burnham K, Anderson D: Model selection and multimodel inference: a practical information-theoretic approach. 2nd edition. New York: Springer; 2003.

82. Schwarz G: Estimating the dimension of a model. Ann Stat 1978 6:461-464

83. Darling AE, Mau B, Perna NT: ProgressiveMauve: multiple genome alignment with gene gain, loss and rearrangement. PLoS One 2010, 5:e11147.

84. Paradis E, Claude J, Strimmer K: APE: Analyses of phylogenetics and evolution in R language. Bioinformatics 2004, 20:289-290.

85. Shimodaira H, Hasegawa M: Multiple comparisons of log-likelihoods with applications to phylogenetic inference. Mol Biol Evol 1999, 16:1114-1116.

86. Shimodaira H, Hasegawa M: CONSEL: for assessing the confidence of phylogenetic tree selection. Bioinformatics 2001, 17:1246-1247.

doi:10.1186/1471-2180-12-220

Cite this article as: Ahlinder et al:: Increased knowledge of Francisella genus diversity highlights the benefits of optimised DNA-based assays. BMC Microbiology 2012 12:220.

\section{Submit your next manuscript to BioMed Central and take full advantage of:}

- Convenient online submission

- Thorough peer review

- No space constraints or color figure charges

- Immediate publication on acceptance

- Inclusion in PubMed, CAS, Scopus and Google Scholar

- Research which is freely available for redistribution 